\title{
Temperature dependence of the training effect in electrodeposited $\mathrm{Co} / \mathrm{CoO}$ nanotubes
}

\author{
M. P. Proenca, ${ }^{1, a)}$ J. Ventura, ${ }^{1}$ C. T. Sousa, ${ }^{1}$ M. Vazquez, ${ }^{2}$ and J. P. Araujo ${ }^{1}$ \\ ${ }^{1}$ IFIMUP and IN-Institute of Nanoscience and Nanotechnology and Dep. Física e Astronomia, \\ Univ. Porto, Rua do Campo Alegre 687, 4169-007 Porto, Portugal \\ ${ }^{2}$ Instituto de Ciencia de Materiales de Madrid, CSIC, 28049 Madrid, Spain
}

(Received 5 June 2013; accepted 12 July 2013; published online 29 July 2013)

\begin{abstract}
High aspect ratio $\mathrm{Co} / \mathrm{CoO}$ nanotubes (NTs) were obtained by potentiostatic electrodeposition of Co inside nanoporous alumina templates followed by the natural oxidation of their inner walls. Magnetic measurements performed at low temperatures after field cooling the samples from above its blocking temperature $\left(T_{\mathrm{B}} \sim 220 \mathrm{~K}\right)$, evidenced the existence of exchange bias (EB) coupling between the $\mathrm{Co}$ ferromagnetic outer wall and the $\mathrm{CoO}$ antiferromagnetic inner wall of the NTs. A decrease in the magnitude of the EB field was measured at $T<T_{\mathrm{B}}$ when cycling the $\mathrm{Co} / \mathrm{CoO}$ NT arrays through consecutive hysteresis loops. This decrease is known as the training effect (TE) and is here studied in the $6 \mathrm{~K} \leq T<T_{\mathrm{B}}$ temperature range. The TE was fitted using the recursive Binek formula, giving small values for the characteristic decay rate of the training behavior, and evidencing a decrease of EB with increasing antiferromagnetic layer thickness. A phenomenological theory for the temperature dependence of the TE in exchange biased systems was applied for the first time to core-shell nanotubular structures. The good agreement obtained between the experimental results and the theoretical data, provided a strong confirmation of the qualitative correctness of the spin configuration relaxation model used in these systems. (C) 2013 AIP Publishing LLC. [http://dx.doi.org/10.1063/1.4816696]
\end{abstract}

\section{INTRODUCTION}

The exchange bias (EB) coupling between a ferromagnetic (FM) and an antiferromagnetic (AFM) layer has been the focus of intense research due to its importance in spintronic and high-density magnetic recording devices. ${ }^{1,2}$ This effect is seen as a shift of the FM hysteresis loop by an EB field $\left(H_{\mathrm{ex}}\right)$ measured from the origin when field cooling the sample from above the Néel temperature $\left(T_{\mathrm{N}}\right)$ of the AFM material. ${ }^{1-5}$ In addition, when cycling the FM/AFM bilayer structure through consecutive hysteresis loops, a $H_{\mathrm{ex}}$ decrease is often observed. This phenomenon, called training effect (TE), can be explained as arising from the partial loss of the AFM net magnetization, as its spin structure rearranges with each magnetization reversal of the FM layer. ${ }^{2,5-8}$ The TE plays a crucial role in the reliable performance of devices based on EB. One should also note that both the EB and its training effect strongly depend on temperature $(T)$, disappearing above the so-called blocking temperature $\left(T_{\mathrm{B}}\right)$ of the AFM material. Therefore, the complete understanding of the $\mathrm{TE}$ of $\mathrm{EB}$ and respective temperature dependence in different AFM/FM geometrical configurations is of extreme importance for further advances in the technological and scientific community.

Since the discovery of $\mathrm{EB}$ in $\mathrm{Co} / \mathrm{CoO}$ particles over 50 years ago, ${ }^{9}$ it has been found in a variety of different systems with FM/AFM interfaces, including core-shell nanoparticles, ${ }^{10-12}$ thin film systems, ${ }^{3,5,13,14}$ lithographed nanostructures, ${ }^{15-18}$ and (more recently) in high aspect ratio

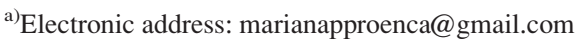

core-shell nanotubes (NTs). ${ }^{19}$ The latter have attracted much interest due to their potential applications in nanoelectronic devices, catalysis, high-density recording media, and drug delivery. ${ }^{20,21}$ Their shape anisotropy combined with tunable nanosized dimensions give rise to new interesting phenomena, such as enhanced magnetic anisotropy and higher coercivity. $^{22}$ In particular, magnetic NTs show great advantages over most EB nanostructures as they present high surface areas, small wall thicknesses and a hollow inner core that can be coated, oxidized, or filled with different materials, increasing the potential applicability of such structures and respective magnetic properties. ${ }^{23,24}$ However, no report was yet published on the temperature dependence of the TE in core-shell FM/AFM nanotubular structures, although its understanding is highly important for the correct implementation of these systems in magnetic devices.

In this work, ordered hexagonal arrays of Co NTs were electrodeposited inside nanoporous alumina templates (NpATs) with diameters of $\sim 40 \mathrm{~nm}$ and interpore distances of $\sim 105 \mathrm{~nm}$. Nanotubular heterostructures of FM/AFM bilayers were obtained after the natural oxidation of the inner Co NT walls, forming Co/CoO NTs. Temperature dependent magnetic measurements allowed us to study the EB phenomenon and the respective TE exhibited by our arrays of tubular core-shell AFM/FM bilayer nanostructures. The EB effect in the fabricated $\mathrm{Co} / \mathrm{CoO}$ NTs was found to arise at temperatures below $220 \mathrm{~K}$, close to the bulk $T_{\mathrm{N}}$ value of $\mathrm{CoO}$ $(293 \mathrm{~K}),{ }^{4,13}$ and in good agreement with reported $T_{\mathrm{B}}$ values for $\mathrm{Co} / \mathrm{CoO}$ heterolayers., ${ }^{1,311,12}$ Training of the EB effect was also measured at several temperatures in the range of $6 \mathrm{~K} \leq T \leq 200 \mathrm{~K}$ and fitted using a recursive formula 
proposed by Binek. ${ }^{6}$ The temperature dependence of the training effect was also obtained and fitted using a theoretical model previously reported for bilayer thin films. ${ }^{25}$

\section{EXPERIMENTAL DETAILS}

NpATs with ordered hexagonal array of pores were prepared by a two-step anodization process of high-purity $\mathrm{Al}$ $(99.999 \%)$ disks in $0.3 \mathrm{M}$ oxalic acid at $40 \mathrm{~V}$ and $\sim 4{ }^{\circ} \mathrm{C}$. ${ }^{26,27}$ The first anodization was performed for $24 \mathrm{~h}$ to improve the hexagonal pattern, while the second anodization lasted $\sim 20 \mathrm{~h}$ leading to a membrane thickness of $\sim 50 \mu \mathrm{m}$. The NpATs obtained with these conditions have ordered hexagonal nanopore arrays with diameters $d \sim 35 \mathrm{~nm}$ and interpore distances of $\sim 105 \mathrm{~nm}$.

After the anodization processes, the NpATs were detached from the substrate by chemically etching the $\mathrm{Al}$, and the pores were opened from both sides of the membrane by removing the alumina layer at the pores' bottom. The opening of the pores was performed by floating the sample in phosphoric acid, which led to a small enlargement of the final pore diameter to $\sim 40 \mathrm{~nm} .{ }^{28}$ For the subsequent potentiostatic electrodeposition of Co NTs inside the pores, an Au metallic contact of $\sim 50 \mathrm{~nm}$ was sputtered at the pores' opened ends to serve as the working electrode during deposition. ${ }^{29} \mathrm{~A} \mathrm{Pt}$ mesh and $\mathrm{Ag} / \mathrm{AgCl}$ (in $4 \mathrm{M} \mathrm{KCl}$ ) were used as counter and reference electrodes, respectively. Further details on the membranes preparation for subsequent electrodeposition of magnetic NTs can be found in Ref. 29.

Cobalt electrodeposition was then performed in an aqueous solution of $0.89 \mathrm{M} \mathrm{CoSO}_{4} \cdot 7 \mathrm{H}_{2} \mathrm{O}$ and $0.49 \mathrm{M} \mathrm{H}_{3} \mathrm{BO}_{3}$, at $30{ }^{\circ} \mathrm{C}$, and applying a constant potential of $-1.5 \mathrm{Vvs}$. $\mathrm{Ag} /$ $\mathrm{AgCl}$ for $2 \mathrm{~min}$, using a Solartron 1480 MultiStat. Morphological characterization was performed using a scanning electron microscope (SEM; FEI Quanta 400FEG). Prior to bottom SEM imaging, ion-milling was performed to remove the Au contact and smooth the NpAT surface. The milling process was carried out using an ion-beam sputter deposition system by Commonwealth Scientific Corporation. ${ }^{30}$ The NpATs filled with Co NTs were then left in air for several months after electrodeposition, to allow natural oxidation of the Co tops/ walls to occur. Due to the highly oxidative nature of the cobalt element, exposing the Co layer to ambient atmosphere leads to the formation of an antiferromagnetic polycrystalline $\mathrm{CoO}$ layer at the surface. ${ }^{31-35}$ In this work the Co NTs were left in air for 9 months, prior to their magnetic characterization, forming an estimated oxide layer thickness of $\sim 4 \mathrm{~nm}$. Temperature (6-250 K) dependent magnetic measurements were performed after field cooling the samples in $50 \mathrm{kOe}$ from above room temperature, using a superconducting quantum interference device (SQUID) MPMS magnetometer from Quantum Design, and with the magnetic field $(H)$ applied perpendicular to the NT axis.

\section{RESULTS AND DISCUSSION}

\section{A. Morphological characterization}

Ordered hexagonal arrays of Co NTs with outer diameters of $\sim 40 \mathrm{~nm}$, wall thicknesses of $\sim 10 \mathrm{~nm}$, intertube distances of $\sim 105 \mathrm{~nm}$ and lengths of $\sim 40 \mu \mathrm{m}$, were successfully obtained by electrodeposition inside NpATs. Figure 1(a) shows a bottom SEM image of the electrodeposited Co NT arrays after ion-milling of the Au working electrode, illustrating the tubular shape and the hollow cores that typically form a NT-like structure. By exposing the NpAT filled with Co NTs to air, natural oxidation of the inner Co NT walls occurs. ${ }^{33,34}$ Figure 1(b) gives a schematic representation of the $\mathrm{Co} / \mathrm{CoO}$ bilayer NTs after natural oxidation of Co. In this work, the oxidation process was performed for 9 months, resulting in $\mathrm{Co} / \mathrm{CoO}$ bilayer NTs with estimated layer thicknesses of $\left(t_{\mathrm{Co}}, t_{\mathrm{CoO}}\right) \sim(6,4) \mathrm{nm}$. The oxide layer thickness is estimated by analysing the decrease in the magnetization values with increasing oxidation times, as will be further seen in Sec. III C. These bilayer tubular nanostructures are thus formed of an outer FM and inner AFM layer, providing a very interesting system to study the training effect of exchange bias.

\section{B. Exchange bias coupling}

$\mathrm{Co} / \mathrm{CoO}$ NTs were previously reported to exhibit a loop shift in both parallel and perpendicular directions of the applied magnetic field, when measuring the magnetic hysteresis loops $[M(H)]$ at $6 \mathrm{~K}$, after field-cooling in $50 \mathrm{kOe}$ from $320 \mathrm{~K}$ (above $T_{\mathrm{N}}^{\mathrm{CoO}} \sim 290 \mathrm{~K}$ ). ${ }^{19}$ This was interpreted as arising from the exchange bias coupling between the outer FM and the inner AFM layers. The shift observed in the $M(H)$ loops along the field axis is defined as the exchange bias field $H_{\mathrm{ex}}=\left(H_{\mathrm{cL}}+H_{\mathrm{cR}}\right) / 2$, where $H_{\mathrm{cL}}$ and $H_{\mathrm{cR}}$ are the left and right coercive fields, respectively. The temperature dependence of EB also allowed the determination of a maximum $T_{\mathrm{B}}$ value of $\sim 220 \mathrm{~K}$, which is $\sim 70 \mathrm{~K}$ below the bulk $T_{\mathrm{N}}^{\mathrm{CoO}} \cdot{ }^{19}$ Additionally, the magnitude of the EB field was found much higher when $H$ was applied perpendicular to the tube axis. Therefore, for the study of the temperature dependence of the TE in the $\mathrm{Co} / \mathrm{CoO}$ NTs presented in this work, the magnetic measurements were performed with $H$ applied only in the perpendicular direction.

\section{Training effect of EB}

The training of the exchange bias field was studied at $T=6,50,125,200$, and $250 \mathrm{~K}$, after field cooling the $\mathrm{Co} /$ $\mathrm{CoO}$ NT arrays in $50 \mathrm{kOe}$ from $320 \mathrm{~K}$ to $T$. Consecutive

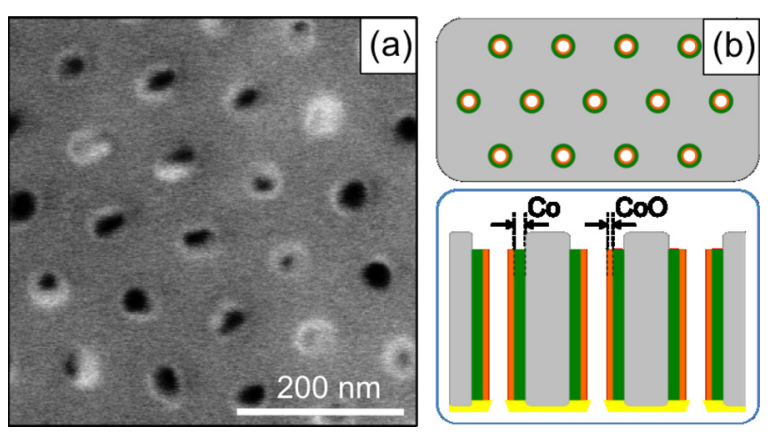

FIG. 1. (a) SEM bottom image of a NpAT filled with Co NTs, after $\sim 200 \mathrm{~nm}$ of etching by ion-milling. (b) Schematic top and cross-sectional representations of a $\mathrm{Co} / \mathrm{CoO} \mathrm{NT}$ array. 

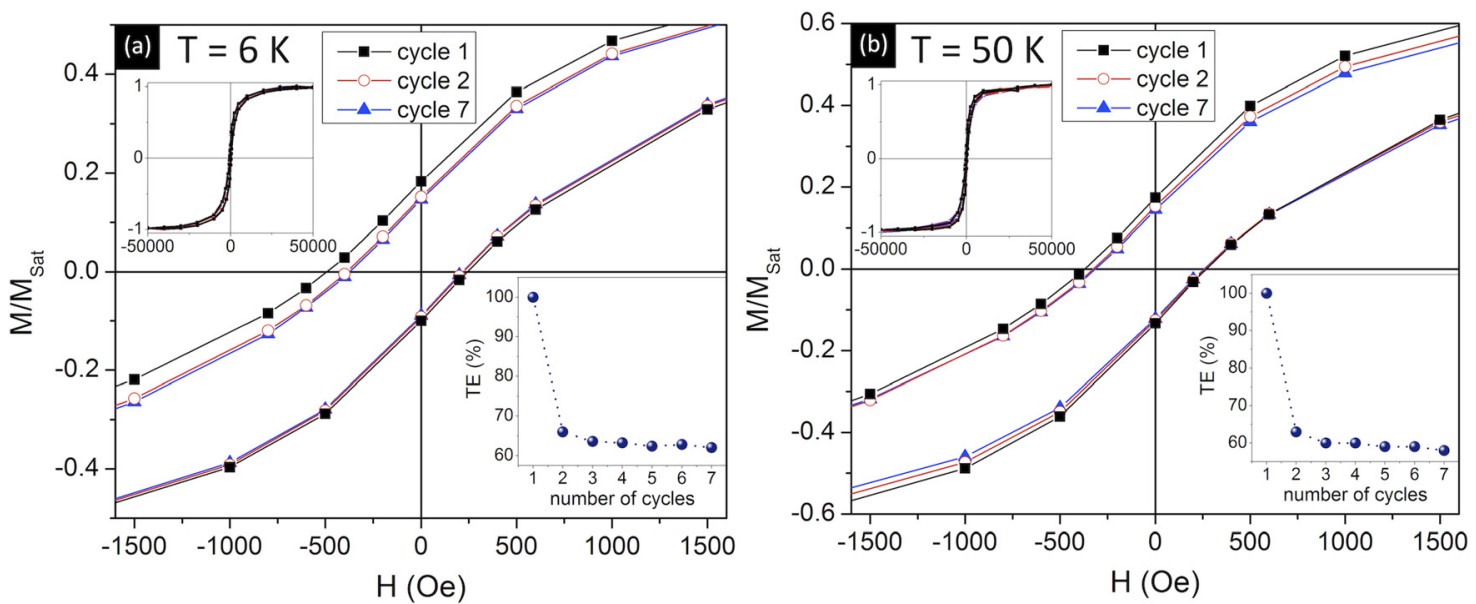

FIG. 2. First, second, and seventh magnetic hysteresis loops of $\mathrm{Co} / \mathrm{CoO} \mathrm{NT}$ arrays measured at (a) $6 \mathrm{~K}$ and (b) $50 \mathrm{~K}$, after field cooling in $50 \mathrm{kOe}$, by applying the magnetic field perpendicular to the NT axis. Upper insets in (a) and (b) show the complete hysteretic cycles, evidencing the saturation of the loops. Lower insets in (a) and (b) show the TE percentage measured at 6 and $50 \mathrm{~K}$, respectively.

magnetic hysteresis loops were then measured with a maximum applied field of $50 \mathrm{kOe}$, and the $\left|H_{\mathrm{ex}}\right|$ values extracted from each $M(H)$. Figure 2 shows the first, second and seventh hysteresis loop for $\mathrm{Co} / \mathrm{CoO}$ NTs measured at $6 \mathrm{~K}$ [Fig. 2(a)] and $50 \mathrm{~K}$ [Fig. 2(b)], evidencing the decrease in the EB field with the number of cycles $(n)$. The $\left|H_{\mathrm{ex}}(n)\right|$ dependence reveals a monotonic decrease of the EB effect when cycling the magnetic field through consecutive loops, corresponding to the training effect (Fig. 3). Since $T_{\mathrm{B}} \sim 220 \mathrm{~K}$ for the $\mathrm{Co} /$ CoO NTs studied, no loop shift was observed at $T=250 \mathrm{~K}$.

The lower insets of Fig. 2 show the percentage of TE measured at $6 \mathrm{~K}$ [Fig. 2(a)] and at $50 \mathrm{~K}$ [Fig. 2(b)] in Co/ $\mathrm{CoO}$ NTs. The TE\% is defined as the relative decrease in $H_{\text {ex }}$ from the first to the $n^{\text {th }}$ cycle $^{2}$

$$
\mathrm{TE}_{n}(\%)=\left(1-\frac{H_{\mathrm{ex}}^{1}-H_{\mathrm{ex}}^{n}}{H_{\mathrm{ex}}^{1}}\right) \times 100(\%)
$$

where $H_{\mathrm{ex}}^{n}$ is the exchange bias field at the $n^{\text {th }}$ cycle. A large decrease of the EB to $\sim 65 \%$ from the first to the second cycles is observed, while for the subsequent cycles EB only drops $\sim 2 \%$. Recent studies attributed the strong $H_{\text {ex }}$ decrease from the first to the second cycle to a non-equilibrium or metastable arrangement of the AFM spins upon field cooling, ${ }^{5,36}$ that could be driven by the symmetry of the AFM anisotropy. ${ }^{8,37}$ The reconfiguration of the AFM spin structure could also be attributed to a reorientation of the AFM domains at the FM/AFM interface during field reversal or domain wall movements. ${ }^{38}$ Other reports considered that AFM clusters having lower anisotropy barriers, or not strongly coupled via exchange interactions to their neighbors, might lose their degree of order upon reversing the field. ${ }^{39}$ When the field is cycled back, the magnetic moment of such spin clusters does not realign into the original configuration due to the absence of further heating. C. Binek explained the TE of EB within the framework of nonequilibrium thermodynamics of the spin configurational relaxation at the AFM surface. ${ }^{6}$ This spin relaxation towards equilibrium was assumed to be driven by the reversal of the FM magnetization during the consecutive cycling of the external magnetic field. Therefore, the gradual decrease of $\left|H_{\text {ex }}\right|$ with $n$ would reflect the rearrangement of the spin structure of the AFM layer after each reversal of the FM layer magnetization. ${ }^{2,5,6,40}$ Based on free energy considerations, C. Binek obtained the following recursive formula to describe TE: ${ }^{6}$

$$
H_{\mathrm{ex}}^{n+1}-H_{\mathrm{ex}}^{n}=-\gamma\left(H_{\mathrm{ex}}^{n}-H_{\mathrm{ex}}^{\infty}\right)^{3},
$$

where $H_{\mathrm{ex}}^{\infty}$ is the exchange bias field in the limit of an infinite number of cycles and $\gamma$ describes the characteristic decay rate of the training behavior. The physical parameter $\gamma$ depends on the leading expansion coefficient $b$ of the free energy, on a factor $\zeta$ which is proportional to the coupling constant between the AFM and FM layer, and on a damping constant $\xi$ that can be considered as a typical inverse relaxation time ${ }^{6}$

$$
\gamma=\frac{b}{\zeta^{2} \xi}
$$

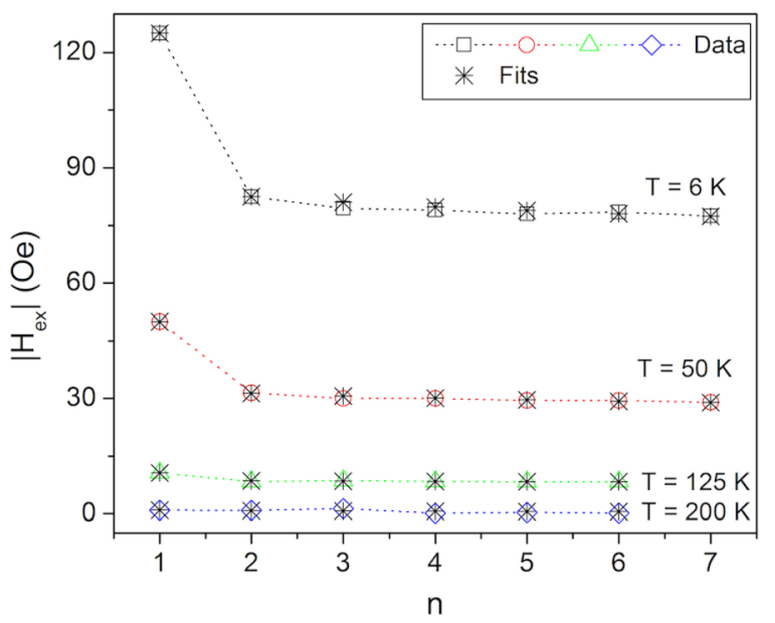

FIG. 3. Dependence of $\left|H_{\mathrm{ex}}\right|$ on the number of magnetic hysteresis cycles (n), measured at $6,50,125$, and $200 \mathrm{~K}$ after field cooling in $50 \mathrm{kOe}$, by applying the magnetic field perpendicular to the tube axis, and respective fits using Eq. (2). 
The results presented in Fig. 3 were fitted using the recursive relation [Eq. (2)], for each individual temperature. Comparing the exchange bias measured at $T=6 \mathrm{~K}$ in the $\mathrm{Co} / \mathrm{CoO}$ NTs presented in this work (after 9 months of oxidation), and in $\mathrm{Co} / \mathrm{CoO}$ NTs with a lower oxidation time (4 months), ${ }^{19}$ a smaller $H_{\text {ex }}$ was found for those that were exposed for longer periods. One should note that, due to the highly oxidative nature of cobalt, an oxide layer of $\sim 2 \mathrm{~nm}$ is expected to be formed after a few days of exposure to ambient atmosphere. ${ }^{31-35}$ Therefore, we estimate the thicknesses of the FM/AFM layers in $\mathrm{Co} / \mathrm{CoO}$ NTs after 4 and 9 months of oxidation as $\left(t_{\mathrm{FM}}, t_{\mathrm{AFM}}\right) \sim(7,3) \mathrm{nm}$ and $\sim(6,4) \mathrm{nm}$, respectively. Such increase in the AFM layer thickness affects the strength of the FM/AFM coupling. In particular, when decreasing $t_{\mathrm{FM}}$ in a FM/AFM bilayer system, an increase in exchange bias would be expected according to

$$
H_{\mathrm{ex}}=\frac{J_{\text {int }}}{M_{\mathrm{FM}} t_{\mathrm{FM}}},
$$

where $J_{\text {int }}$ is the interface coupling constant, and $M_{\mathrm{FM}}$ is the saturation magnetization of the FM layer. ${ }^{19}$ However, our results show that $H_{\mathrm{ex}}$ decreases with decreasing $t_{\mathrm{FM}}$. According to Eq. (4), this is only possible if compensated by a decrease in the coupling energy. This was also confirmed by the lower $\gamma$ value obtained for the sample with 4 months of oxidation $\left(\gamma \sim 2.3 \times 10^{-6} \mathrm{Oe}^{-2}\right)$ when compared to that of the $\mathrm{Co} / \mathrm{CoO}$ NTs after 9 months of oxidation $\left(\gamma \sim 1.7 \times 10^{-4} \mathrm{Oe}^{-2}\right)$. The $\gamma$ values are proportional to the inverse square of the coupling constant between the FM and AFM layers. Therefore, the observed increase of $\gamma$ with $t_{\mathrm{AFM}}$ for FM/AFM nanotubular structures can be interpreted as a decrease in the coupling constant between the AFM and FM layers when increasing the AFM layer thickness. Previous reports on the $\mathrm{TE}$ in $\mathrm{Co} / \mathrm{CoO}$ thin films with different $\mathrm{AFM}$ thicknesses have also shown the same tendency of EB to decrease with increasing $t_{\mathrm{AFM}} \cdot{ }^{38,41}$ In particular, thicker AFM layers were found to be more stable, thus leading to a smaller TE. ${ }^{38}$

\section{Temperature dependence of TE}

To study the temperature dependence of the TE of EB in $\mathrm{Co} / \mathrm{CoO} \mathrm{NT}$ arrays, we analyzed in detail the variation of $H_{\text {ex }}$ for consecutive $M(H)$ cycles. Figure 3 shows the experimental data of $H_{\mathrm{ex}}$ versus $n$ (opened symbols) and the corresponding results of the best fits of Eq. (2) (stars), at different temperatures. The two-parameter fits return the values of $\gamma$ and $H_{\mathrm{ex}}^{\infty}$, which in turn are used to calculate the theoretical data from the recursive formula in Eq. (2). The inset of Fig. 4 shows the expected tendency of $H_{\mathrm{ex}}^{\infty}$ to increase with decreasing temperature.

To better understand the thermal evolution of the TE in EB heterostructures, Binek et al. developed a phenomenological theory that allowed its explicit determination in terms of $\gamma=\gamma(T){ }^{25}$ The model of Binek et al. uses a discretized Landau-Khalatnikov equation, where the training effect is considered within the framework of relaxation phenomena. ${ }^{25}$ In this model, the reversal of the FM layer is the driving

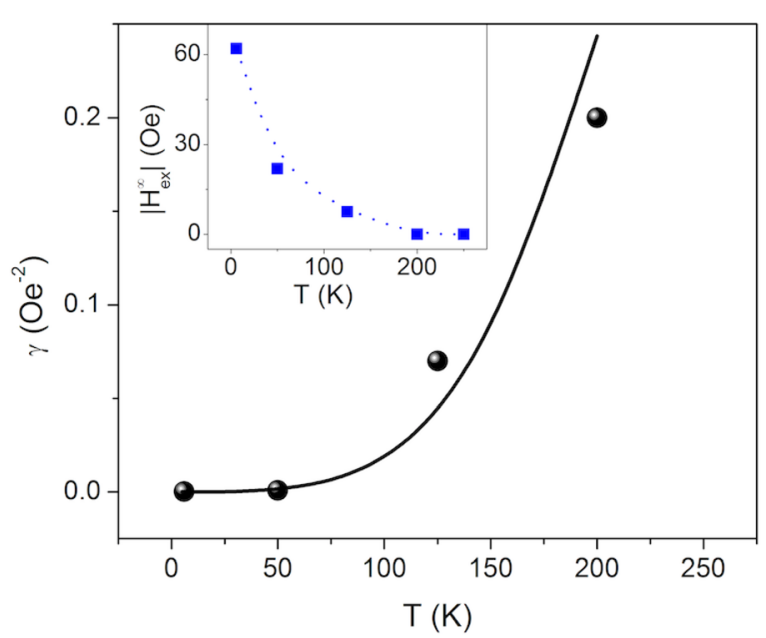

FIG. 4. Temperature dependence of the $\gamma$ parameter and corresponding fit (line) using Eqs. (5) and (6). Inset shows the temperature dependence of the $H_{\mathrm{ex}}^{\infty}$ parameter.

force that leads the AFM interfacial magnetization towards equilibrium. Considering a first-order approximation of the mean-field theory, Binek et al. obtained the following expression: ${ }^{25}$

$$
\gamma(T)=C\left(\frac{\eta_{e}(T) \tanh \left[\frac{T_{N} \eta_{e}(T)}{T}\right]}{T\left\{T\left(1+\cosh \left[\frac{2 T_{N} \eta_{e}(T)}{T}\right]\right)-2 T_{N}\right\}}\right)^{2},
$$

where $\mathrm{C}$ is a free fitting parameter that summarizes several phenomenological parameters, and $\eta_{e}(T)$ can be approximated by ${ }^{25}$

$$
\eta_{e}(T) \approx \tanh \left[\frac{T_{N}}{T} \sqrt{\frac{3\left(T_{N}-T\right)}{T_{N}}}\right]
$$

The combination of Eqs. (5) and (6) provides an explicit fitting function for the experimental values of $\gamma(T)$. One should note that, in the studied system, the temperature at which the EB disappears corresponds to the blocking temperature, $T_{\mathrm{B}}$, and thus $T_{\mathrm{N}}$ should be replaced by $T_{\mathrm{B}}$ in the previous equations. Figure 4 shows the $\gamma(T)$ data (dots) obtained from the best fit of $H_{\mathrm{ex}}(n)$, for each individual temperature, using Eq. (2). The line corresponds to the best fit obtained using Eqs. (5) and (6), confirming the qualitative correctness of the spin configurational relaxation model. Additionally, the good quality fit obtained for $\gamma(T)$ in the studied $\mathrm{Co} / \mathrm{CoO} \mathrm{NT}$ arrays, is a further proof that the this model can be successfully used to describe the temperature dependence of the TE of EB in different geometrical configurations of bilayer exchange bias nanostructures.

\section{CONCLUSIONS}

The temperature dependence of the training effect of exchange bias was studied for the first time in $\mathrm{Co} / \mathrm{CoO}$ nanotube arrays. Isothermal training effects were measured by consecutive cycling magnetic hysteresis loops at different 
temperatures $(6 \leq T \leq 200 \mathrm{~K})$, and extracting the values of the exchange bias field as a function of $n$. The TE was then fitted using the recursive Binek formula, providing an estimation for the temperature dependence of the characteristic decay rate of the training behavior (fitting parameter $\gamma$ ). A theoretical model previously reported for the temperature dependence of the TE in bilayer thin films was applied for the first time to core-shell nanotubular structures. The good agreement found between the experimental results and the theoretical data, indicates the qualitative accuracy of the spin configuration relaxation model used in these systems.

\section{ACKNOWLEDGMENTS}

M.P.P. and C.T.S. are thankful to FCT for post-doctoral grants SFRH/BPD/84948/2012 and SFRH/BPD/82010/2011, respectively. J.V. acknowledges financial support through FSE/POPH. M.V. thanks the Spanish Ministry of Economia y Competitividad, MEC, under project MAT2010-20798C05-01. The authors acknowledge funding from FCT under project PTDC/FIS/098943/2008 and through the Associated Laboratory - IN.

${ }^{1}$ J. Nogues and I. K. Schuller, J. Magn. Magn. Mater. 192, 203 (1999).

${ }^{2}$ J. Ventura, J. P. Araujo, and J. B. Sousa, Phys. Rev. B 77, 184404 (2008).

${ }^{3}$ S. Brems, D. Buntinx, K. Temst, C. Van Haesendonck, F. Radu, and H. Zabel, Phys. Rev. Lett. 95, 157202 (2005).

${ }^{4}$ T. Maurer, F. Zighem, F. Ott, G. Chaboussant, G. Andre, Y. Soumare, J.-Y. Piquemal, G. Viau, and C. Gatel, Phys. Rev. B 80, 064427 (2009).

${ }^{5}$ S. R. Ali, M. R. Ghadimi, M. Fecioru-Morariu, B. Beschoten, and G. Guntherodt, Phys. Rev. B 85, 012404 (2012).

${ }^{6}$ C. Binek, Phys. Rev. B 70, 014421 (2004).

${ }^{7}$ S. Brems, D. Buntinx, K. Temst, and C. Van Haesendonck, Phys. Rev. Lett. 99, 067201 (2007).

${ }^{8}$ V. Baltz, Appl. Phys. Lett. 102, 062410 (2013).

${ }^{9}$ W. H. Meiklejohn and C. P. Bean, Phys. Rev. 102, 1413 (1956).

${ }^{10}$ M. P. Proenca, C. T. Sousa, A. M. Pereira, P. B. Tavares, J. Ventura, M. Vazquez, and J. P. Araujo, Phys. Chem. Chem. Phys. 13, 9561 (2011).

${ }^{11}$ M. Feygenson, Y. Yiu, A. Kou, K.-S. Kim, and M. C. Aronson, Phys. Rev. B 81, 195445 (2010).

${ }^{12}$ M. P. Fernandez-Garcia, P. Gorria, M. Sevilla, A. B. Fuertes, R. Boada, J. Chaboy, G. Aquilantid, and J. A. Blanco, Phys. Chem. Chem. Phys. 13, 927 (2011).
${ }^{13}$ S. Maat, K. Takano, S. S. P. Parkin, and E. E. Fullerton, Phys. Rev. Lett. 87, 087202 (2001).

${ }^{14}$ E. Shipton, K. Chan, T. Hauet, O. Hellwig, and E. E. Fullerton, Appl. Phys. Lett. 95, 132509 (2009).

${ }^{15}$ V. Baltz, J. Sort, S. Landis, B. Rodmacq, and B. Dieny, Phys. Rev. Lett. 94, 117201 (2005).

${ }^{16}$ D. Tripathy, A. O. Adeyeye, N. Singh, and R. L. Stamps, Nanotechnology 20, 015304 (2009).

${ }^{17}$ D. Tripathy and A. O. Adeyeye, J. Appl. Phys. 105, $07 \mathrm{C} 110$ (2009).

${ }^{18}$ S. Laureti, S. Y. Suck, H. Haas, E. Prestat, O. Bourgeois, and D. Givord, Phys. Rev. Lett. 108, 077205 (2012).

${ }^{19}$ M. P. Proenca, J. Ventura, C. T. Sousa, M. Vazquez, and J. P. Araujo, Phys. Rev. B 87, 134404 (2013).

${ }^{20}$ S. S. P. Parkin, M. Hayashi, and L. Thomas, Science 320, 190 (2008).

${ }^{21}$ L. Zhang, T. Petit, K. E. Peyer, and B. J. Nelson, Nanomedicine: Nanotechnol. Biol. Med. 8, 1074 (2012).

${ }^{22}$ N. Ahmad, J. Y. Chen, J. Iqbal, W. X. Wang, W. P. Zhou, and X. F. Han, J. Appl. Phys. 109, 07A331 (2011).

${ }^{23}$ S. J. Son, X. Bai, A. Nan, H. Ghandehari, and S. B. Lee, J. Controlled Release 114, 143 (2006).

${ }^{24}$ M. P. Proenca, C. T. Sousa, J. Ventura, J. P. Araujo, J. Escrig, and M. Vazquez, SPIN 2, 1250014 (2012).

${ }^{25}$ C. Binek, X. He, and S. Polisetty, Phys. Rev. B 72, 054408 (2005).

${ }^{26} \mathrm{H}$. Masuda and K. Fukuda, Science 268, 1466 (1995).

${ }^{27}$ M. P. Proenca, C. T. Sousa, J. Ventura, M. Vazquez, and J. P. Araujo, Electrochim. Acta 72, 215 (2012).

${ }^{28}$ M. P. Proenca, C. T. Sousa, J. Escrig, J. Ventura, M. Vazquez, and J. P. Araujo, J. Appl. Phys. 113, 093907 (2013).

${ }^{29}$ M. P. Proenca, C. T. Sousa, J. Ventura, M. Vazquez, and J. P. Araujo, Nanoscale Res. Lett. 7, 280 (2012).

${ }^{30}$ D. C. Leitao, J. Ventura, C. T. Sousa, J. M. Teixeira, J. B. Sousa, M. Jaafar, A. Asenjo, M. Vazquez, J. M. De Teresa, and J. P. Araujo, Nanotechnology 23, 425701 (2012).

${ }^{31}$ B. H. Miller and E. Dan Dahlberg, Appl. Phys. Lett. 69, 3932 (1996).

${ }^{32}$ F. Radu, M. Etzkorn, T. Schmitte, R. Siebrecht, A. Schreyer, K. Westerholt, and H. Zabel, J. Magn. Magn. Mater. 240, 251 (2002).

${ }^{33}$ S. Sahoo, S. Polisetty, Y. Wang, T. Mukherjee, X. He, S. S. Jaswal, and C. Binek, J. Phys.: Condens. Matter 24, 096002 (2012).

${ }^{34}$ L. Smardz, U. Kbbler, and W. Zinn, J. Appl. Phys. 71, 5199 (1992).

${ }^{35}$ M. Verelst, T. O. Ely, C. Amiens, E. Snoeck, P. Lecante, A. Mosset, M. Respaud, J. M. Broto, and B. Chaudret, Chem. Mater. 11, 2702 (1999).

${ }^{36}$ A. G. Biternas, R. W. Chantrell, and U. Nowak, Phys. Rev. B 82, 134426 (2010).

${ }^{37}$ A. Hoffmann, Phys. Rev. Lett. 93, 097203 (2004).

${ }^{38}$ A. G. Biternas, U. Nowak, and R. W. Chantrell, Phys. Rev. B 80, 134419 (2009).

${ }^{39}$ B. Kaeswurm and K. O’Grady, Appl. Phys. Lett. 99, 222508 (2011).

${ }^{40}$ Z. M. Tian, J. T. Chen, S. L. Yuan, Y. S. Zhang, Z. Z. Ma, H. N. Duan, and C. L. Lu, J. Appl. Phys. 110, 103902 (2011).

${ }^{41}$ U. Nowak, A. Misra, and K. D. Usadel, J. Appl. Phys. 89, 7269 (2001). 